\title{
Thinking Globally, Acting Locally: On the Issue of Training Set Imbalance and the Case for Local Machine Learning Models in Chemistry
}

\author{
Mojtaba Haghighatlari, ${ }^{1, *}$ Ching-Yen Shih, ${ }^{1}$ and Johannes Hachmann ${ }^{1,2,3, \dagger}$ \\ ${ }^{1}$ Department of Chemical and Biological Engineering, University at Buffalo, \\ The State University of New York, Buffalo, NY 14260, United States \\ ${ }^{2}$ Computational and Data-Enabled Science and Engineering Graduate Program, \\ University at Buffalo, The State University of New York, Buffalo, NY 14260, United States \\ ${ }^{3}$ New York State Center of Excellence in Materials Informatics, Buffalo, NY 14203, United States
}

The appropriate sampling of training data out of a potentially imbalanced data set is of critical importance for the development of robust and accurate machine learning models. A challenge that underpins this task is the partitioning of the data into groups of similar instances, and the analysis of the group populations. In molecular data sets, different groups of molecules may be hard to identify. However, if the distribution of a given data set is ignored then some of these groups may remain under-represented and the sampling biased, even if the size of data is large. In this study, we use the example of the Harvard Clean Energy Project (CEP) data set to assess the challenges posed by imbalanced data and the impact that accounting for different groups during the selection of training data has on the quality of the resulting machine learning models. We employ a partitioning criterion based on the underlying rules for the CEP molecular library generation to identify groups of structurally similar compounds. First, we evaluate the performance of regression models that are trained globally (i.e., by randomly sampling the entire data set for training data). This traditional approach serves as the benchmark reference. We compare its results with those of models that are trained locally, i.e., within each of the identified molecular domains. We demonstrate that local models outperform even the best global models by considerable margins and are more efficient in their training data needs. We propose a strategy to redesign training sets for the development of improved global models. While the resulting uniform training sets can successfully yield robust global models, we identify the distribution mismatch between feature representations of different molecular domains as a critical limitation for any further improvement. We take advantage of the discovered distribution shift and propose an ensemble of classification and regression models to achieve generalized and reliable models across the CEP data set. This study provides a benchmark for the development of future methodologies concerned with imbalanced chemical data.

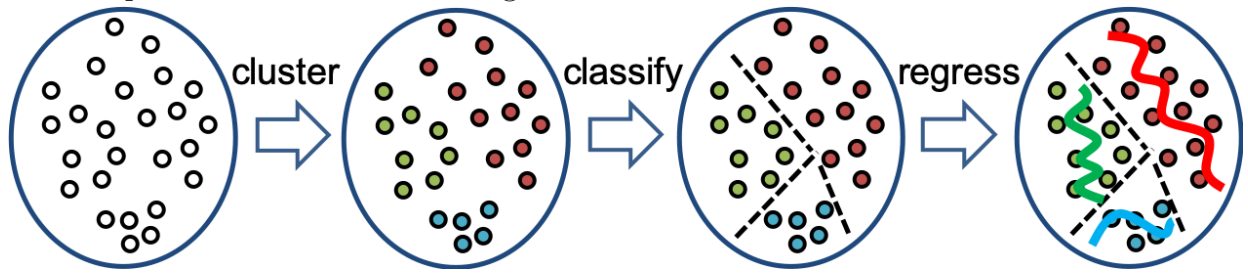

\section{INTRODUCTION}

Machine learning (ML) is in the process of revolutionizing several aspects of chemical (and materials) research. ML approaches illuminate underlying patterns in chemical data, they facilitate efficient predictions in the characterization and behavior of chemical systems, and they augment conventional processes of decision making in chemical research $[1,2]$. One application of ML is the creation of data-derived surrogate models that accelerate the intensive process of molecular discovery, design, and development by orders of magnitude $[3,4]$. The application of ML on the results of virtual high-throughput screening (HTPS) studies has been one of the earliest and most successful approaches for the large-scale exploration of molecular space [5-8].

\footnotetext{
* mojtabah@buffalo.edu

† hachmann@buffalo.edu
}

A majority of methodological advancements for ML in the chemical domain have so far focused on improving the performance of data-derived prediction models for desirable materials properties. Research on other pertinent questions of chemical data mining and modeling has received less attention. Examples of these issues are: (i) diversity/sparsity of the molecular structures, (ii) applicability domains of trained ML models, and (iii) learning from imbalanced data. While these issues require expertise from domain sciences [9], they are ultimately interconnected and share common solutions across disciplines. In this work, we address these challenges on a well-known molecular data set.

A data set is imbalanced if it can be partitioned into groups of similar instances (e.g., molecules), but the count of instances per group differs significantly [10]. By that means, even if the data set is large, some groups of may remain underrepresented (i.e., minority groups). Typically, ML approaches fail to capture the characteristics of the minority groups because they are less ex- 
posed to the instances they contain. The primary challenge in many molecular data sets is the lack of unique or even rigorous criteria to discover the underlying population manifold. Prior knowledge, chemical intuition, or a direct mapping between structural features and groups (e.g., via clustering, i.e., an unsupervised ML approach) can achieve this task, but the distribution may also remain unknown.

The trend in chemical and materials studies goes towards developing models that are as general as possible and that can thus cover the entire molecular space. However, there are also studies that argue against the pursuit of universal prediction models. For instance, a study by Goldsmith et al. proposed the method of subgroup discovery to cluster the crystal structures of semiconductors [11]. The results of their work show that local models trained on subgroups of the entire compound data - can significantly outperform a global model that is trained on the natural distribution of the overall data set. More recently, Kailkhura et al. introduced an ML framework that successfully addresses the skewed distribution of materials by partitioning their target property space [12]. While this approach has similar objectives to our work, we believe that the imbalance in data is more due to structural features rather than their properties. The exploration of molecular space using ML models is subject to the applicability domain of those models, which is restricted by the sparsity of the initial training data. We thus believe that taking the locality of data into account can have tremendous benefits, e.g., in the context of HTPS results $[13,14]$.

To exemplify, visualize, and address the challenge of imbalanced data in a molecular system, we focus on the Harvard Clean Energy Project (CEP) data set [15]. CEP is one of the prominent HTPS efforts to find organic photovoltaics for their application in solar cells. This data set has been widely used in several modeling and method development projects [16-19], mainly to recover the rigorous and deterministic quantum chemical mapping from the structure/topology of a molecule to its properties. However, all of these ML approaches focus on the natural distribution of the data and lead to global models that ignore the adverse impacts of the imbalanced chemical structures in the data set. In this study, we take advantage of the imposed initialization and constraints in the combinatorial exploration of molecular space (e.g., by HTPS approaches) to improve the data-driven predictive performance further. We show this extra patternrecognized information leads to reliable prediction across the data population, and thus enhances the applicability domain of ML models and enables us to better understand the structure-property relationships. In a broader perspective, the proposed approaches attempt to asset and establish efficient practices to develop generalized predictive models for organic molecular data sets.

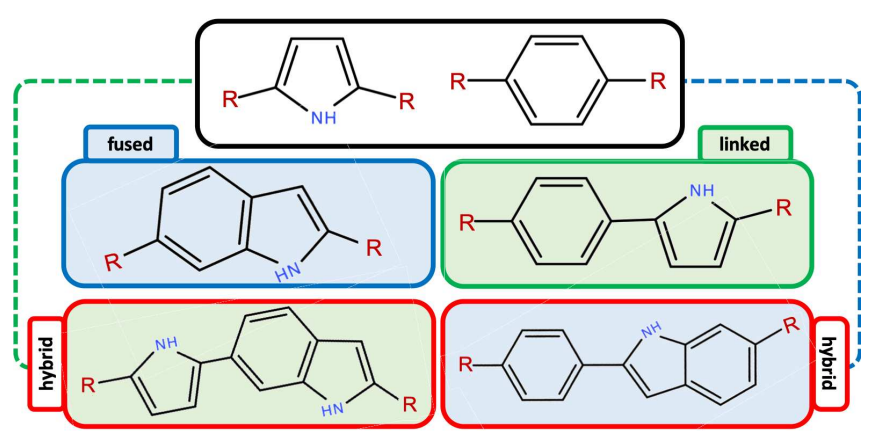

FIG. 1: Examples of fused, linked, and hybrid molecules initiated from two building blocks (i.e., benzene and pyrrole) with two reaction sites (' $R$ 's represent chemical handles) per each. The product of reaction also has two chemical handles to participate in the next reactions and create larger molecules. A hybrid molecule is a product of linking a building block to a fused molecule, or vice versa.

\section{BACKGROUND, METHODS, AND COMPUTATIONAL DETAILS}

\section{A. Motivation}

As described by Hachmann et al. [20], the CEP molecular library is generated using 26 distinct building blocks that react with each other and create new fragments based on two types of reactions (i.e., linking or fusion). The maximum number of building blocks per molecule is limited to five. Although all the 26 building blocks are prevalent substructures for the photovoltaic applications, the type of reaction between them results in very different molecular moieties that might not be feasible to synthesis. The motivation for this work is based on the feedback from experimental collaborators regarding the ease of synthesis for molecules that are only a product of linking reactions. The synthesis condition for fused fragments are often harsh and may negatively result in a ring expansion or contraction.

Thus, the initial goal of this study is to interpret the generation of each molecule in the CEP library based on the applied combination rules and types of building blocks. We divide molecules in the CEP data set to three groups of fused, linked, or hybrid. As it is illustrated in Fig. 1, a hybrid molecule is a result of both linking and fusion reaction between constituent building blocks. Note that the reaction scheme has not been captured initially along with the original CEP library generation. One immediate solution to this problem is to search for all possible combinations of building blocks in the molecules. However, this is an intractable approach due to the large number of possible combinations. Therefore, we develop an algorithm to perform this task and will discuss details in Sec. II B. 
After extracting the substructure information, our motivation for the rest of the study is two-fold. First, we investigate the distribution of top candidates in the subgroups of the molecules. We train ML regression models on each subgroup separately (i.e., build local models) and compare their performance with the model that is trained on a random sample of the entire CEP data set (i.e., a global model). Second, we utilize the extra information regarding the distribution of clusters in the data set to improve the performance of the global model. For this purpose, we oversample under-represented subgroups and create a uniform distribution of clusters in the training set. We observe that the main challenge for any further improvements in the global model is the shift in the distribution of the feature representation for each class of molecules. Therefore, we apply classification and feature transformation methods to demonstrate the impact of the distribution mismatch in the training sets. This extra approach leads us towards an ensemble of classification and regression models to generalize well from minority subgroups without compensation from the majority subgroups. Thus, the central claim of this study is to provide the most accurate and generalized ML model for predicting photovoltaic properties of the molecules in the CEP data set. In summary, the contribution of this paper is as follows:

- We propose an algorithm to exploit the structure of the CEP data set. This approach is based on the reaction scheme that molecules have undertaken during the library generation.

- When the reaction scheme is identified, we partition the entire CEP data set based on the synthesis feasibility, thereby, we achieve benchmark data with identified subgroups to assess the underrepresentation of similar molecular structures.

- We next investigate the effect of the imbalanced classes on the performance of ML models that are developed using pure random sampling of the entire data set. Subsequently, we achieve computationally efficient ML models that outperform the state-ofthe-art predictive models for the CEP data set.

- Finally, we automatize the entire approach by redesigning the ML training sets and training a classification model to alleviate the side effect of distribution shift for regression models.

\section{B. CEP Data Set and Molecular Characterization}

The CEP data set contains more than 2.3 million organic photovoltaic molecules that are candidates for donor materials in the solar cells. The target property to evaluate the performance of those solar cells is the power conversion efficiency (PCE). The PCE values are approximated using Scharber model and electronic properties of donor molecules (i.e., molecules in the CEP data set). The electronic properties are calculated at BP86/def2SVP [21-23] level of the Kohn-Sham density functional theory $[24,25]$. All the molecules are represented using SMILES strings, which provide 2D information of the molecular structures, that is, atom type and connectivity.

We propose an algorithm based on the molecular graphs to characterize the unique combination scheme that has been undertaken for the generation of each molecule in the CEP library. The algorithm is specific to the CEP data set and takes advantage of the heterocyclic structure of the building blocks. In the following we describe the overall pseudo-algorithm:

1. represent each molecule as a graph of nodes and edges and keep track of their corresponding chemical labels (i.e., atom and bond types).

2. identify all the cycles in the molecular graphs with size less than 6 . They correspond to the 5- and 6membered molecular rings in the structure of building blocks.

3. look for nodes that are shared between the rings. These types of nodes represent the fusion reaction between rings.

4. look for edges that are not involved in any of the rings. These types of edges form the linking connection between rings.

5. discover type of building blocks and their connections based on atom and bond types and the unique combination of rings in building blocks.

The second step of the algorithm requires an efficient code for finding loops of specific sizes and not bigger than that. The available algorithms for this step, e.g., Johnston algorithm, are computationally expensive. We use the built-in function available in the OpenBable package [26], which can efficiently perform the first two steps of the algorithm. The final result of this substructure analysis represents the exact type, order, and symmetry of building blocks based on the position of reaction sites in each molecule. This substructure information could be further utilized to focus on a group of molecular moieties that may be of higher importance for the rational design of materials.

Note that we use the term cluster to distinguish molecules in the CEP data set that have been generated using only fusion reaction (i.e., fused cluster), only linking reaction (i.e., linked cluster), or a combination of both (i.e., hybrid cluster). The choice of this terminology is not entirely arbitrary; it tends to differentiate this task from the supervised classification approach. More importantly, it has an analogy with unsupervised clustering approach that will be discussed in our following publications as an automated and generalized fashion to distinguish organic molecules. Thus in this study, we 
undertake a clustering approach based on the chemical intuitions from and only for CEP data set.

\section{Machine Learning Details}

The ML task in this study is of supervised learning category due to the availability of the labeled data. A supervised learning approach can be considered as a function that maps the input features to the target outputs [27]. If the output labels have continuous numerical values, the supervised learning task is a regression problem, and if the labels are distinct and finite (categorical), the problem is classification. Thus in this study, we train a regression model to predict the scalar PCE values and a classification model to label each molecule as a fused, linked, or hybrid cluster. We carry out the classification problem to evaluate the distribution shift in the input features of the three clusters. This method enables us to understand the structural diversity of the data based on the feature representation of the molecules in each of the clusters. We also apply principal component analysis to reduce the dimension of the feature space for the visualization of the distribution shift.

We use deep neural networks (DNN) for both of the regression and classification tasks [28, 29]. The DNN consists of neurons that behave as a simple feature transformation unit. Originally, each neuron sends out the result of an activation function acting on the total sum of the weighted inputs that receives from all connected neurons. The neurons are organized in consecutive layers and may be partially connected. Thus, the entire DNN model is able to transform the input features to the latent space, where the mapping to the target output becomes linear. We train a fully-connected standard architecture of DNN with three hidden layers in this work. We optimize other hyper-parameters (e.g., activation functions, regularization parameter, learning rate, etc.) using the 10 -fold cross-validation approach on the $90 \%$ of the data as the training set. The remaining $10 \%$ are held out for the final evaluation of the model. We use a genetic algorithm to efficiently search and optimize the hyperparameter space. In addition to the cross-validation, we carry out two additional approaches to avoid over-fitting: (1) the regularization term to penalize the parameters that are learning the noise [30], and (2) the early stopping approach, which stops training iterations when the model improvement is negligible. Both of these methods avoid unnecessary model complexity.

We perform this ML workflow using ChemML $[1,31$, 32], our program package for machine learning and informatics in chemical and materials research. In this work, Chem $M L$ employs the Keras library [33] with Tensorflow backend [34] to develop the DNN models. The scikitlearn library provides tools for data preprocessing and model evaluation [35]. To plot learning curves, we select five different subset sizes spaced uniformly over the range of the training set size. We next average the training and evaluation of the ML models over five different random sampling with each of the five subset sizes. The main evaluation metric for regression models is the mean absolute error (MAE). MAE represents the deviation of the predicted values from the target properties. For classification models, we use the ratio of correct predictions to evaluate the accuracy of our classifiers.

\section{Feature Representation}

In the cheminformatics and materials informatics, the input features for an ML model are called descriptors [36]. Descriptors provide a numerical representation of the molecules and are the most important aspect of the ML models. A number of studies present that substructurebased descriptors provide essential representation to predict several properties of molecules [37]. This type of descriptors, which are also known as the molecular fingerprint, indicate the presence or absence of particular substructures in the molecule. In this study, we use 2048-bit, radius 3 Morgan fingerprint (MFP) [38, 39], from RDKit cheminformatics library [40], as previously recommended in similar studies (concerning the structure and property of molecules) [16].

In addition to Morgan fingerprint, we also use neural fingerprint (NFP) as the state-of-the-art neural network architecture, originally developed and tested on the CEP data set [17]. Previous research has established that NFPs are able to provide the most comprehensive representation of the structural makeups. The NFP take advantage of the flexibility in the design of deep learning architecture. NFPs can be considered as a stand-alone ML model by addition of fully connected layers. Therefore, for the purpose of comparison, we add the same standard neural network that was described in Sec. II C to complete the NFP model. Various versions of similar deep learning architectures have been developed recently $[19,41,42]$. However, the representational limitations that they try to address are not a source of differentiation in the aforementioned clusters of the CEP molecular library. Thus, the NFP is a sufficiently complex and accurate model to serve as the state-of-the-art technique in this study. These models are computationally more demanding than the standard DNN by a factor of 100 in terms of minute calculation on the same computation resource.

\section{RESULTS AND DISCUSSION}

\section{A. Statistical Analysis of the Clusters}

Tab. I shows the statistical analysis of all three clusters in the CEP data set with a focus on the power conversion efficiency (PCE) of organic solar cells. The distribution of PCE values among each cluster clearly shows the linked 
molecules are more favorable for the photovoltaic applications with higher mean positive PCE compared to the other two clusters.

In addition to the analysis of the PCE values, we note that the three clusters are not uniformly distributed. The fused and linked molecules occupy less than $4 \%$ and $24 \%$ of the entire data set, respectively. In the ML literature, these two clusters are known to be under-represented in comparison with the hybrid cluster with close to $74 \%$ of the total data [43]. Therefore, a global ML model trained on a random selection of the CEP data is mostly biased to the hybrid molecules. Since our goal for the rest of the study is to compare the performance of different ML models trained on the three clusters with respect to the global model, we fix the training set size to 50,000 for all training sets. This number fulfills both the minimum number of data points in a cluster and the size of the training data in the recent ML studies on the CEP data set.

TABLE I: Statistical analysis for each cluster in the CEP data set. We obtain the population of each cluster in the entire set of 2.3 million molecules and in a subset of the data with positive PCE. We then compute the average and mean absolute deviation (MAD) for each subset. The population is in million and the avg and

MAD of PCE are in \%.

\begin{tabular}{l|c|ccc}
\hline \hline & all & \multicolumn{3}{c}{$P C E>0$} \\
& population(m) & population(m) & avg(\%) & MAD(\%) \\
\hline fused & $0.07(3 \%)$ & 0.04 & 2.18 & 1.34 \\
linked & $0.54(23 \%)$ & 0.27 & 3.24 & 2.09 \\
hybrid & $1.72(74 \%)$ & 1.07 & 2.61 & 1.60 \\
\hline all & 2.33 & 1.38 & 2.72 & 1.70 \\
\hline \hline
\end{tabular}

Furthermore, Fig. 2 shows the distribution of PCE in the entire CEP data set and also in each of the clusters. The inset violin plot also shows the distribution of the molecules with $\mathrm{PCE}>8 \%$ and reveals the prevalence of linked molecules among top candidates, specificaly those with PCE $>10 \%$. Thereby, a focus on the linked molecules not only improves the synthesis feasibility of molecules but potentially leads towards more useful candidates in photovoltaic materials. Note that the negative PCE is the artifact of the Scharber model and does not present any physical meaning. However, we keep the entire range of the PCE values for the purpose of the training and do not change them to zero. This way we preserve the continuity of the values, which enables the ML model to recover the actual Scharber model.

The other point to attention is that distinguishing the three clusters from each other based on the PCE values is not possible since their distributions approximately overlap. This point implies the fact that sometimes molecular structures are indistinguishable based on their target properties and thus, other criteria are required to categorize them.

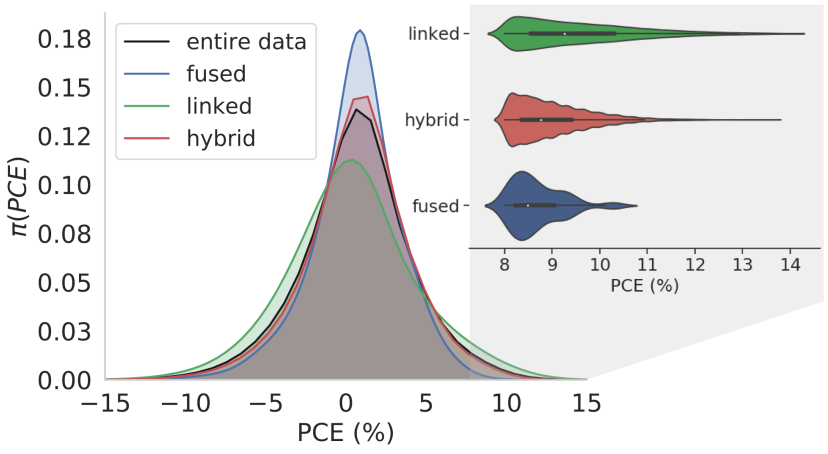

FIG. 2: The histogram showing the distribution of Scharber power conversion efficiency (PCE) values in the entire and each cluster of the CEP data set. The inset violin plot presents the mean, standard deviation and $25 / 75$ th percentile over candidates with $\mathrm{PCE}>8.0 \%$.

\section{B. Predicting PCE of Organic Solar Cells}

Following the statistical analysis of the clusters and based on the size cap of the smallest subgroup (i.e., fused molecules), we randomly select 50,000 data points from each of the clusters and one from the entire CEP data set. The resulting four subsets are referred to as fused, linked, hybrid and random samples. These four samples serve as our data sets for the training and testing of our models in the rest of the study. We next train one standard DNN regression model on each of the training sets and evaluate the model on all the four test sets. We refer to the fused, linked, and hybrid models as local models. Same way, the model for the random sample is called a global model because it can potentially predict the PCE for molecules from any of the clusters. Fig. 3.a shows the absolute deviation of the ML predicted PCE values from Scharber model estimates. As expected, the plot shows that each local model performs better on the test set from the same category of training data. The highest errors belong to the fused and linked models when they are evaluated on test sets from each other's test sets. However, the fused model performs better on the hybrid test set compared to the linked model. This point can also be confirmed based on the comparison between the performance of the hybrid model on the fused and linked test sets. These results determine that hybrid molecules have more in common with fused molecules rather than linked ones. More interestingly, all three local models outperform the random model for prediction on their own type of molecules. The closest performance to the random model belongs to the hybrid model since hybrid molecules are over-represented in the random sample. For a similar reason, the performance of the random model on the fused and linked test sets are significantly worse than their local models. Although more than $70 \%$ of the random sample consists of hybrid molecules, the random 
(a)

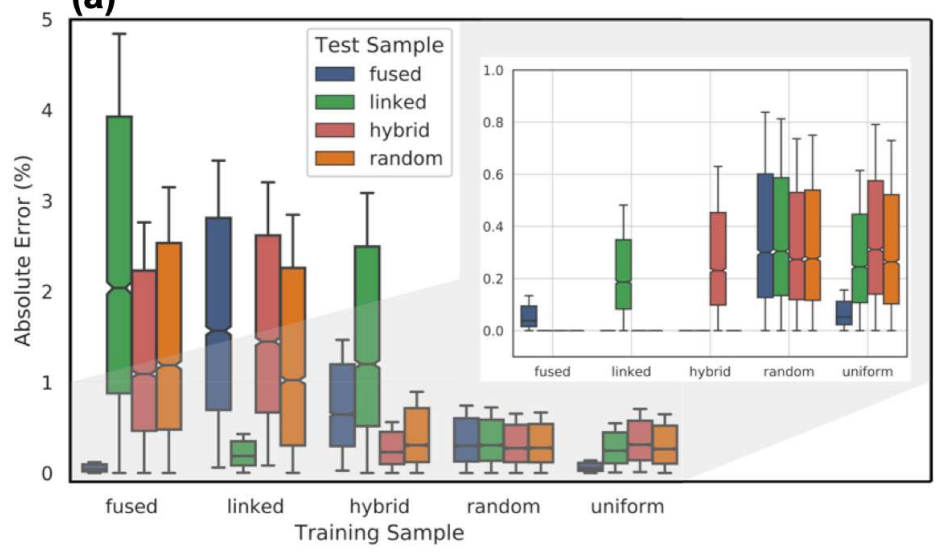

(b)

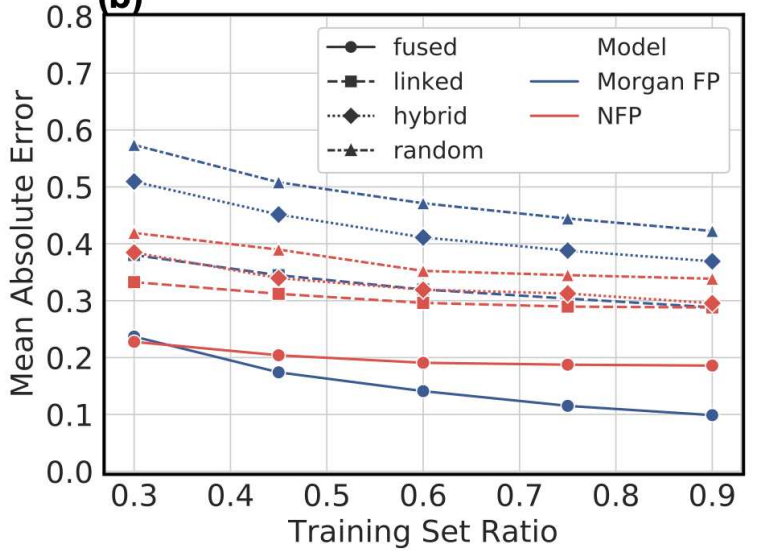

FIG. 3: The performance of standard deep neural network (DNN) regression models for the prediction of power conversion efficiency values in each of the samples. (a) The box plot showing the distribution of the absolute error of predicted PCE values in terms of median, 25/75th percentile and confidence interval. The inset plot highlights all the box plots that present less than 1\% absolute error. (b) The learning curves show the dependency of developed models to the training set size. The blue curves belong to the standard deep neural network trained on Morgan fingerprint (FP) representation. The red curves belong to the neural fingerprint (NFP) model. The mean absolute error is calculated based on the evaluation of each model on the test set from the same sample.

model still performs slightly worse than the model that is trained on $100 \%$ hybrid molecules (i.e., hybrid model).

In addition, Fig. 3b presents the learning curves that are trained and evaluated on each of the samples individually. The dependency of the models to the size of the training set is generally accepted in any ML efforts, and here we see the same trend for all the four samples, as well. The performance of the local models also follows a similar relative trend on the entire range of the training set size. We also note that the risk of bias can be assessed for the fused model once it is trained on $90 \%$ of the fused sample. The reason is that the fused cluster is the smallest cluster in the data and the choice of 50,000 random sample almost covers the entire category. Thus, the ML model has an easy task to predict the properties of outof-sample molecules because it has been exposed to the majority of them from the entire distribution. However, the learning curve for the fused model shows that even $30 \%$ of the fused sample can train a better model than $90 \%$ of the random sample for the prediction of PCE for fused molecules.

All these results confirm that training a model on a random sample is mostly in favor of hybrid molecules that are over-represented in the data set. Note that a better performance of the local models compared to the random model is generally expected. It is well known that ML models intrinsically have better performance for the interpolation tasks rather than extrapolation. Therefore, developing a local model on the portion of data that are of similar characteristics, results in a better performance. However, this point is subject to the clustering approach that lend confidence to the similar characteristics of the subgroups of the molecules. In other words, an arbitrary clustering of a data set that does not emphasize more compelling characteristics of the structures may not reproduce the same results.

We also plot the distribution of the predictions errors across the range of PCE values for each of the test samples. As it is illustrated in Fig. 4, the quality of the predictions for the linked and fused models are evenly distributed over the range of the PCE values. This is another advantage of the suggested clustering scheme that leads to molecular candidates, which are: (i) feasible to synthesis, (ii) more desirable with respect to the target property, (iii) homogeneously represented and thus are easier to model, and (iv) approximately equally representative of the remoter but more desirable range of $\mathrm{PCE}$ values.

\section{The Impact of Clusters on the State-of-the-art Models}

In this section, we use NFP to benchmark the results of the standard DNN models with the state-of-the-art neural network architecture. The learning curve for the NFP model is presented in Fig. 5. The figure is generated based on the same method as described in the previous section. We also merge it with Fig. 3b for an easier comparison. We first confirm that all the training curves are showing a lower MAE by increasing the training set size. The comparison of the learning curves for two fingerprint models on the random sample clearly shows the reason why we call these models as state-ofthe-art. More than 20\% improvement in the prediction accuracy across the random training set is the main rea- 


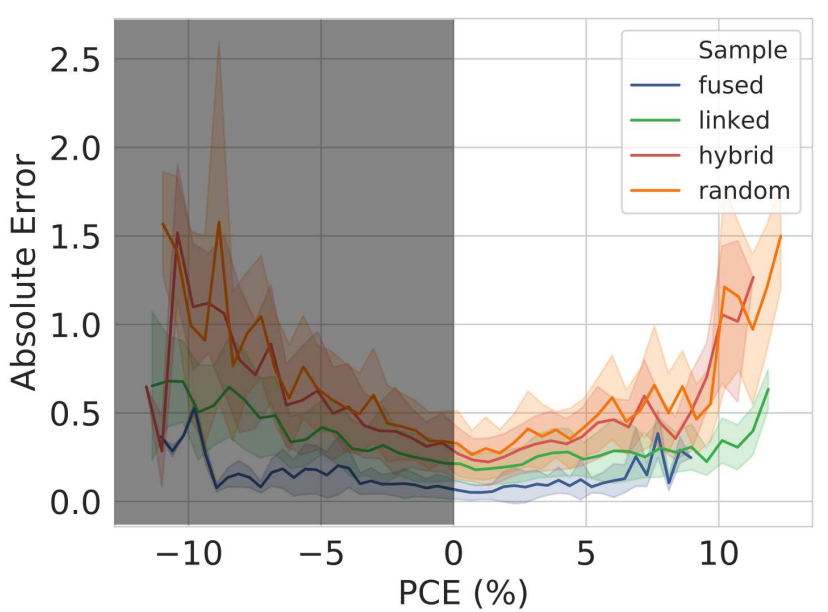

FIG. 4: The distribution of the regression model prediction errors with respect to the PCE values for each of the four samples. The negative PCE values are artifact of the Scharber model, and thus, are faded out.

son that these models have truly increased the excitement in the field. Similar behavior can also be observed for the hybrid sample. However, the linked and fused samples do not exhibit a big difference for the training of the two fingerprint models. The key observation is that for the local models the NFP becomes saturated by adding more training data and stops showing the expected improvement. One reason for such behavior is the structural diversity in the four samples of the data. The random and hybrid molecules not only contain a diverse set of molecules based on the applied reaction rules between building blocks but also are a small portion of many molecules available in their category. The underlying point for the current research is that the significantly more expensive complex models sound ineffective for the prediction of the properties of linked molecules. This point can be counted as the advantage of our clustering approach.

\section{Uniform Oversampling of the Training Data}

One immediate solution to address the issue with imbalanced data is oversampling of the underrepresented clusters. Since enough training data is available for each of the clusters, we simply stack their training sets from the samples as mentioned earlier. Thus, compared to the previous section, a three times bigger training set is now available to train a standard DNN, but with a uniform distribution of clusters. The model will be further evaluated on the same test sets from the four samples. The result is illustrated in Fig. 3a as the uniform training sample. We observe that the model performs better on all the test sets compared to the random sample, except for the hybrid test set. However, the performance of

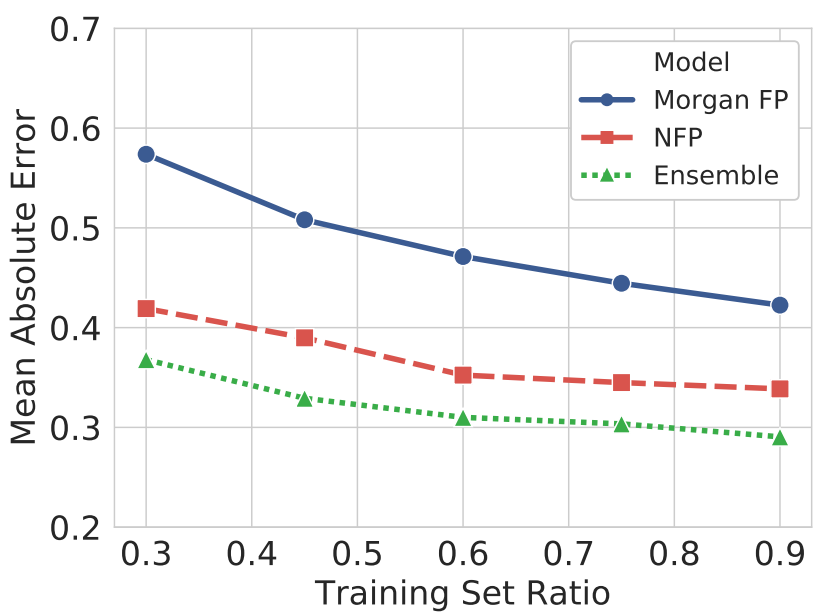

FIG. 5: The learning curves show the decay in mean absolute error of three machine learning (ML) models by increasing the training set size. The blue and red curves belong to the standard neural network and neural fingerprint (NFP) models that are trained on random sample, respectively. The green learning curve presents the proposed ensemble method by merging classification and regression models that are trained and evaluated on three local samples.

the uniform model is not better than the models purely trained on each of the samples. A focus on the performance of the models on the hybrid test set suggests that combination of the clusters do not help our model (i.e., the standard DNN) with respect to the choice of descriptor (i.e., the Morgan fingerprint). This point was also observed by comparing the performance of random and hybrid models on the hybrid test set. We also note that creating three times bigger training set should result in a better performance for the model. However, the performance of our models on the hybrid test set decreases from the hybrid model (trained on $100 \%$ hybrid data) to random model (trained on $70 \%$ hybrid data), and finally to the uniform model (trained on $33 \%$ hybrid data). All in all, the uniform model outperforms the random model generally, and particularly on the fused and linked test sets. Because a focus on the linked molecules is the primary goal of this work, providing a global model that performs better on the linked cluster and globally is considered as a successful outcome.

Based on these results, we discuss that addition of the data from different clusters confuses the model from learning/distinguishing the characteristics of a single cluster. We can explain this point better with the concept of the distribution shift in the feature representation of the data. For doing so, we first transform the high-dimensional representation (i.e., 2,048 fingerprint vector) to two reduced features by applying principal component analysis (PCA) on 3,000 randomly selected instances of the uniform training set. The scatter plot 


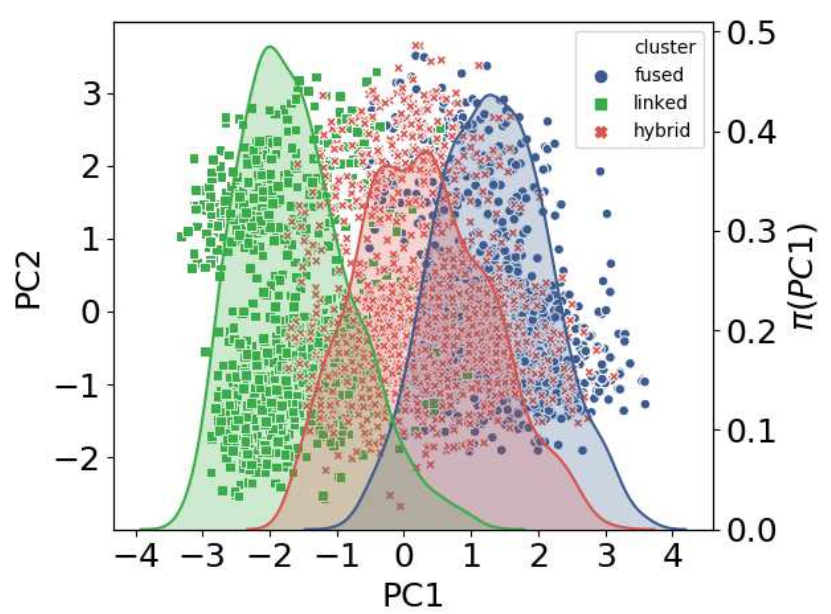

FIG. 6: The scatter plot shows the first and second principal components of a uniform distribution of three clusters specified with color codes. The distribution of first principal component is also plotted accordingly for each cluster. The density of the probabilities are on the right y-axis.

of the data points with their color-coded cluster labels is shown in Fig. 6. The distribution of the data from each cluster clearly illustrates the distribution shift between the clusters. The results show that the linked and fused clusters are very different in terms of the fingerprint representation. Moreover, the distribution of the fused and hybrid principal components has a more significant overlap than the linked and hybrid distributions. All these outcomes are along with our discussions in Sec. IIIB as well.

\section{E. Ensemble Learning by Combining Regression and Classification Models}

As we discussed in the previous section, a simple linear PCA is able to capture the main structural difference between the three clusters. Thereby, our choice of feature representation can linearly distinguish the three suggested categories of molecules. We also should note that all these molecules share the same building blocks in their structures. However, distinguishing the three clusters based on their connections is an entirely different task from the prediction of target properties (i.e., PCE values). In fact, a clustering approach only removes the extra degree of freedom (i.e., the connection between building blocks), and let the local regression models focus on the most distinguishing structural makeups of the molecules. Thus, for developing a global model that has similar performance with our best local models, we propose an ensemble method by merging the classification and regression models.

In this section, we train a classifier on the same uni- form sample that was created in Sec. IIID. The classifier should be able to label an unseen molecule as fused, linked, or hybrid category. We then use our best local regression models accordingly to predict the PCE that corresponds to that molecule. We optimize a standard DNN model to classify the three clusters. The only difference between our classification and regression models is the choice of activation function for the last layer, which is the Softmax function here instead of a linear function for regression. The uniform training set is used for developing the model because it is a fairly balanced data set. We also change the training set size to assess the performance of the classifiers on the tiny portion of the data (e.g., 0.001 ratio that corresponds to 135 data points). All the models are evaluated on the same test sets of the initial four samples, and the resulting learning curves are shown in Fig. 7. The figure shows that even with less than 2,000 uniform sample of the CEP data, we can get a model with $98 \%$ accuracy to classify three clusters of molecules. The performance of the model on the test sets deteriorates in the order of linked, fused, and then hybrid test sets. These results are also in common with Fig. 6, because the feature representation of hybrid molecules is distributed between two other clusters and has a more considerable overlap with fused molecules. The underlying point is that the linked cluster is highly distinguishable from the other two clusters. Thus, we can also examine the distribution shift in the structural features of molecules based on the performance of a classification task. We further extend the work by merging the classification model and our best local regression models to develop a global predictive model for the CEP data set. This approach has been long known as ensemble learning method and boosts the overall performance of the models that share same hypothesis space. The green curve in Fig. 5 presents the learning curve for the ensemble model, evaluated on the random test set. The MAE for ensemble model is lower than both of the Morgan and NFP models across the training set ratio. Ta. II summarizes the lowest MAE for the three models. The deep ensemble learning approach presents $31 \%$ and $15 \%$ improvement compared to the Morgan fingerprint and NFP, respectively. Besides, for the linked molecules, the performance of the three models are similar and approximately show similar amount of improvement with respect to their random models. It should be noted that the accuracy of classification model for the linked molecules is close to $100 \%$ and thus, the performance of the ensemble model for the linked cluster is very similar to the linked models.

\section{CONCLUSIONS}

In the work presented here, we introduced a structurebased partitioning scheme for molecular data sets that allows us to identify different domains in compound space. We showed the benefits of creating local ML models that 
TABLE II: The prediction error of three regression models in terms of mean absolute error (MAE) \pm standard deviation. The table summarizes Fig. 5 at $90 \%$ training set ratio.

\begin{tabular}{l|ccc}
\hline \hline & \multicolumn{3}{|c}{$M A E(\%)$} \\
& Morgan FP & NFP & Ensemble \\
\hline linked & $0.288 \pm 0.003$ & $0.288 \pm 0.002$ & - \\
random & $0.423 \pm 0.004$ & $0.340 \pm 0.004$ & $0.290 \pm 0.005$ \\
\hline \hline
\end{tabular}

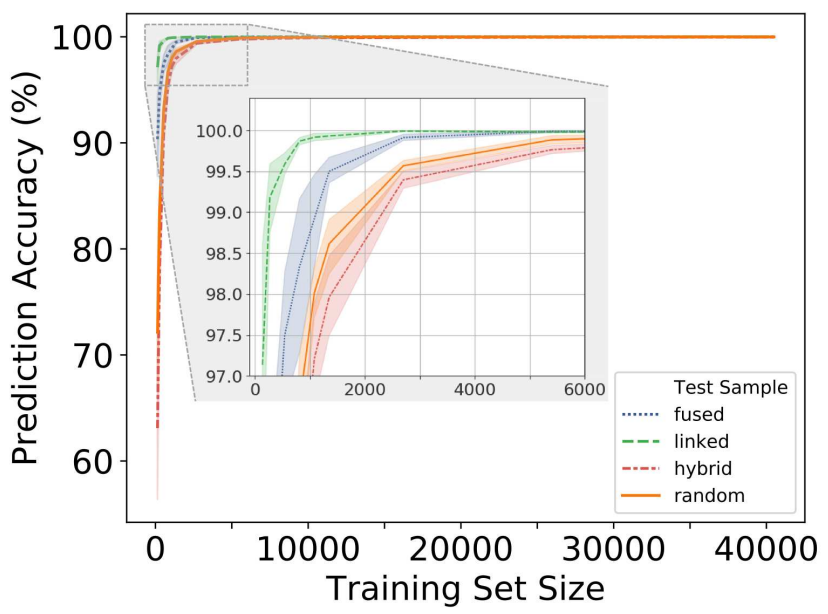

FIG. 7: We develop a model to classify three clusters of the molecules based on the training on the uniform sample of the data. The learning curves showing the prediction accuracy of the classifier with respect to the training set size, and evaluation on four test samples. The inset plot focus on the turning point of the learning curves.

take advantage of the distinct nature of these domains compared to a single global model that does not account for their differences. The improvements in performance and efficiency are considerable, and even standard ML models outperform the most advanced (and correspondingly demanding), state-of-the-art ML approaches. Another attractive feature our study revealed is that local models exhibit a more uniform performance across the spectrum of target property values, including the desirable extremes, for which global models tend to degrade. However, the principal bottleneck for developing local models is the size cap of the minority domains.

We also advanced the use of a classification model and the idea of ensemble learning to achieve the performance of local models but for global predictions. Our statistical analysis of the data set and its imbalance suggests that the latter should be tackled by focusing on the choice of the feature representation, as the sparsity in a feature space can adversely affect a regression task. We propose to resolve this issue by breaking down the imposed sparseness using clustering or classification techniques. Beyond to cluster-aware regression approach presented in this paper, we are currently pursuing an automated process that includes the utilization of unsupervised ML techniques, along with the incorporation (or extraction) of physical priors.

\section{SUPPLEMENTARY MATERIAL}

Electronic supplementary material accompanies this paper and is available through the journal website free of charge. It provides statistical analysis of all data sets that are used in this study (Table S1), and tuned hyperparameter values for trained models (Table S2). We also give a link to the repository that data sets are deployed.

\section{COMPETING FINANCIAL INTERESTS}

The authors declare to have no competing financial interests.

\section{ACKNOWLEDGMENTS}

This work was supported by the National Science Foundation (NSF) CAREER program (grant No. OAC1751161), and the New York State Center of Excellence in Materials Informatics (grants No. CMI-1140384 and CMI-1148092). Computing time on the highperformance computing clusters 'Rush', 'Alpha', 'Beta', and 'Gamma' was provided by the UB Center for Computational Research (CCR). The work presented in this paper is part of MH's PhD dissertation [44]. MH gratefully acknowledges support by Phase-I and Phase-II Software Fellowships (grant No. ACI-1547580-479590) of the National Science Foundation (NSF) Molecular Sciences Software Institute (grant No. ACI-1547580) at Virginia Tech $[45,46]$. We are grateful to Prof. Chong Cheng for valuable discussions and insights.

We gratefully acknowledge the use of data from the Clean Energy Project Database, which was generate with generous support from the IBM World Community Grid (WCG) and the computing time donations from its volunteer participants. Additional information about the project as well as links to join the WCG and the Clean Energy Project can be found in Refs. [47] and [48].
[1] Johannes Hachmann, Mohammad Atif Faiz Afzal, Mojtaba Haghighatlari, and Yudhajit Pal, "Building and deploying a cyberinfrastructure for the data-driven design of chemical systems and the exploration of chemical 
space," Molecular Simulation 44, 921-929 (2018).

[2] Johannes Hachmann, Theresa L. Windus, John A. McLean, Vanessa Allwardt, Alexandra C. SchrimpeRutledge, Mohammad Atif Faiz Afzal, and Mojtaba Haghighatlari, Framing the role of big data and modern data science in chemistry, Tech. Rep. (2018).

[3] Keith T. Butler, Daniel W. Davies, Hugh Cartwright, Olexandr Isayev, and Aron Walsh, "Machine learning for molecular and materials science," Nature 559, 547555 (2018).

[4] Mojtaba Haghighatlari and Johannes Hachmann, "Advances of machine learning in molecular modeling and simulation," Curr. Opin. Chem. Eng. 23, 51-57 (2019).

[5] Geoffroy Hautier, "Finding the needle in the haystack: Materials discovery and design through computational ab initio high-throughput screening," Computational Materials Science 163, 108 - 116 (2019).

[6] Roberto Olivares-Amaya, Carlos Amador-Bedolla, Johannes Hachmann, Sule Atahan-Evrenk, Roel S. Sánchez-Carrera, Leslie Vogt, and Alán AspuruGuzik, "Accelerated computational discovery of highperformance materials for organic photovoltaics by means of cheminformatics," Energy \& Environmental Science 4, 4849-4861 (2011).

[7] Carlos Amador-Bedolla, Roberto Olivares-Amaya, Johannes Hachmann, and Alán Aspuru-Guzik, "Organic Photovoltaics," in Informatics for materials science and engineering: Data-driven discovery for accelerated experimentation and application, edited by Krishna Rajan (Amsterdam: Butterworth-Heinemann, 2013) Chap. 17, pp. 423-442.

[8] Benjamin Sanchez-Lengeling and Alán Aspuru-Guzik, "Inverse molecular design using machine learning: Generative models for matter engineering," Science 361, 360365 (2018).

[9] Mojtaba Haghighatlari, Gaurav Vishwakarma, Mohammad Atif Faiz Afzal, and Johannes Hachmann, "A Physics-Infused Deep Learning Model for the Prediction of Refractive Indices and Its Use for the Large-Scale Screening of Organic Compound Space," ChemRxiv , 1-9 (2019).

[10] Nathalie Japkowicz, "Learning from Imbalanced Data Sets: A Comparison of Various Strategies," Proceeding of Association for the Advancement of Artificial Intelligence 1, 111-117 (2000).

[11] Bryan R. Goldsmith, Mario Boley, Jilles Vreeken, Matthias Scheffler, and Luca M. Ghiringhelli, "Uncovering structure-property relationships of materials by subgroup discovery," New Journal of Physics 19 (2017).

[12] Bhavya Kailkhura, Brian Gallagher, Sookyung Kim, Anna Hiszpanski, and T. Yong-Jin Han, "Reliable and Explainable Machine Learning Methods for Accelerated Material Discovery," Prepr. https//arxiv.org/abs/1901.02717, 1-24 (2019).

[13] Mohammad Atif Faiz Afzal, Mojtaba Haghighatlari, Sai Prasad Ganesh, Chong Cheng, and Johannes Hachmann, "Accelerated Discovery of High-Refractive-Index Polyimides via First-Principles Molecular Modeling, Virtual High-Throughput Screening, and Data Mining ," The Journal of Physical Chemistry C 123, 14610-14618 (2019).

[14] Mohammad Atif Faiz Afzal, Aditya Sonpal, Mojtaba Haghighatlari, Andrew J. Schultz, and Johannes Hachmann, "A Deep Neural Network Model for Pack- ing Density Predictions and its Application in the Study of 1.5 Million Organic Molecules," ChemRxiv , 8217758 (2019).

[15] Johannes Hachmann, Roberto Olivares-amaya, Sule Atahan-Evrenk, Carlos Amador-Bedolla, Roel S. Sanchez-Carrera, Aryeh Gold-Parker, Leslie Vogt, Anna M. Brockway, and Alán Aspuru-Guzik, "The Harvard Clean Energy Project: Large-Scale Computational Screening and Design of Organic Photovoltaics on the World Community Grid," Journal of Physical Chemistry Letters 2, 2241-2251 (2011).

[16] Edward O. Pyzer-Knapp, Kewei Li, and Alán AspuruGuzik, "Learning from the Harvard Clean Energy Project: The Use of Neural Networks to Accelerate Materials Discovery," Advanced Functional Materials 25, 6495-6502 (2015).

[17] David Duvenaud, Dougal Maclaurin, Jorge AguileraIparraguirre, Rafael Gómez-Bombarelli, Timothy Hirzel, Alán Aspuru-Guzik, and Ryan P Adams, "Convolutional Networks on Graphs for Learning Molecular Fingerprints," in proceeding of Advances in Neural Information Processing Systems (2015) pp. 2224-2232.

[18] Hanjun Dai, Bo Dai, and Le Song, "Discriminative Embeddings of Latent Variable Models for Structured Data," Proc. 33rd International Conference on Machine Learning 48 (2016).

[19] Truong Son Hy, Shubhendu Trivedi, Horace Pan, Brandon M. Anderson, and Risi Kondor, "Predicting molecular properties with covariant compositional networks," Journal of Chemical Physics 148 (2018).

[20] Johannes Hachmann, Roberto Olivares-Amaya, Adrian Jinich, Anthony L. Appleton, Martin A. Blood-Forsythe, László R. Seress, Carolina Román-Salgado, Kai Trepte, S. Atahan-Evrenk, Süleyman Er, Supriya Shrestha, Rajib Mondal, Anatoliy Sokolov, Zhenan Bao, and Alán Aspuru-Guzik, "Lead candidates for high-performance organic photovoltaics from high-throughput quantum chemistry-the Harvard Clean Energy Project," Energy and Environmental Science 7, 698-704 (2014).

[21] John P. Perdew, "Density-functional approximation for the correlation energy of the inhomogeneous electron gas," Physical Review B 33, 8822-8824 (1986).

[22] A. D. Becke, "Density-functional exchange-energy approximation with correct asymptotic behavior," Physical Review A 38, 3098-3100 (1988).

[23] Florian Weigend, Reinhart Ahlrichs, K. A. Peterson, T. H. Dunning, R. M. Pitzer, and A. Bergner, "Balanced basis sets of split valence, triple zeta valence and quadruple zeta valence quality for $\mathrm{H}$ to $\mathrm{Rn}$ : Design and assessment of accuracy," Physical Chemistry Chemical Physics 7, 3297 (2005).

[24] R.G. Parr and Y. Weitao, Density-Functional Theory of Atoms and Molecules, International Series of Monographs on Chemistry (Oxford University Press, 1994).

[25] Wolfram Koch and Max C Holthausen, A chemist's guide to density functional theory (John Wiley \& Sons, 2015).

[26] Noel M O'Boyle, Michael Banck, Craig A James, Chris Morley, Tim Vandermeersch, and Geoffrey R Hutchison, "Open Babel: An open chemical toolbox," Journal of Cheminformatics 3, 33 (2011).

[27] Matthias Rupp, O. Anatole Von Lilienfeld, and Kieron Burke, "Guest Editorial: Special Topic on Data-Enabled Theoretical Chemistry," Journal of Chemical Physics 148 (2018), 10.1063/1.5043213, arXiv:1806.02690. 
[28] Jrgen Schmidhuber, "Deep learning in neural networks: An overview," Neural Networks 61, 85 - 117 (2015).

[29] Adam C. Mater and Michelle L. Coote, "Deep Learning in Chemistry," Journal of Chemical Information and Modeling 59, 2545-2559 (2019).

[30] F. Girosi, M. Jones, and T. Poggio, "Regularization theory and neural networks architectures," Neural Computation 7, 219-269 (1995).

[31] Mojtaba Haghighatlari, Gaurav Vishwakarma, Doaa Altarawy, Ramachandran Subramanian, Bhargava Urala Kota, Aditya Sonpal, Srirangaraj Setlur, and Johannes Hachmann, "ChemML: A Machine Learning and Informatics Program Package for the Analysis, Mining, and Modeling of Chemical and Materials Data," ChemRxiv , 8323271 (2019).

[32] Mojtaba Haghighatlari, Gaurav Vishwakarma, Doaa Altarawy, Ramachandran Subramanian, Bhargava Urala Kota, Aditya Sonpal, Srirangaraj Setlur, and Johannes Hachmann, "ChemML - A Machine Learning and Informatics Program Package for the Analysis, Mining, and Modeling of Chemical and Materials Data," (2019).

[33] François Chollet, "Keras, available at https://keras.io," (2015).

[34] Abadi Martin, Agarwal Ashish, Barham Paul, Brevdo Eugene, Chen Zhifeng, Citro Craig, Corrado Greg S., Davis Andy, Dean Jeffrey, Devin Matthieu, Ghemawat Sanjay, Goodfellow Ian, Harp Andrew, Irving Geoffrey, Isard Michael, Yangqing Jia, Jozefowicz Rafal, Kaiser Lukasz, Kudlur Manjunath, Levenberg Josh, Mané Dandelion, Monga Rajat, Moore Sherry, Murray Derek, Olah Chris, Schuster Mike, Shlens Jonathon, Steiner Benoit, Sutskever Ilya, Talwar Kunal, Tucker Paul, Vanhoucke Vincent, Vasudevan Vijay, Viégas Fernanda, Vinyals Oriol, Warden Pete, Wattenberg Martin, Wicke Martin, Yu Yuan, and Zheng Xiaoqiang, "\{TensorFlow $\}$ : Large-Scale Machine Learning on Heterogeneous Systems, available at https://www.tensorflow.org," (2015).

[35] F Pedregosa, G Varoquaux, A Gramfort, V Michel, B Thirion, O Grisel, M Blondel, P Prettenhofer, R Weiss, V Dubourg, J Vanderplas, A Passos, D Cournapeau, M Brucher, M Perrot, and E Duchesnay, "Scikit-learn: Machine Learning in Python," Journal of Machine Learning Research 12, 2825-2830 (2011).

[36] O. Anatole Von Lilienfeld, "Quantum machine learning in chemical compound space," Angewandte Chemie International Edition 57, 4164-4169 (2018).

[37] Rampi Ramprasad, Rohit Batra, Ghanshyam Pilania, Arun Mannodi-Kanakkithodi, and Chiho Kim, "Ma- chine learning in materials informatics: recent applications and prospects," npj Computational Materials 3, 54 (2017).

[38] Harry L Morgan, "The Generation of a Unique Machine Description for Chemical Structures-A Technique Developed at Chemical Abstracts Service." Journal of Chemical Documentation 5, 107-113 (1965).

[39] David Rogers and Mathew Hahn, "ExtendedConnectivity Fingerprints," Journal of Chemical Information and Modeling 50, 742-754 (2010).

[40] Gregory Landrum, "RDKit: Open-source cheminformatics, avaialble at http://www.rdkit.org," (2006).

[41] Justin Gilmer, Samuel S Schoenholz, Patrick F Riley, Oriol Vinyals, and George E Dahl, "Neural Message Passing for Quantum Chemistry," in Proceeding of 34th International Conference on Machine Learning, Vol. 70 (JMLR.org, 2017) pp. 1263-1272.

[42] Kristof. T. Schütt, Huziel E. Sauceda, Pieter-Jan Kindermans, Alexandre Tkatchenko, and Klaus-Robert Müller, "Schnet : A deep learning architecture for molecules and materials," The Journal of Chemical Physics 148, 241722 (2018).

[43] Nicolae C. Iovanac and Brett M. Savoie, "Improved Chemical Prediction from Scarce Data Sets via Latent Space Enrichment," The Journal of Physical Chemistry A 123, 4295-4302 (2019).

[44] Mojtaba Haghighatlari, Making Machine Learning Work in Chemistry: Methodological Innovation, Software Development, and Application Studies, Ph.D. thesis, University at Buffalo (2019).

[45] Anna Krylov, Theresa L. Windus, Taylor Barnes, Eliseo Marin-Rimoldi, Jessica A. Nash, Benjamin Pritchard, Daniel G.A. Smith, Doaa Altarawy, Paul Saxe, Cecilia Clementi, T. Daniel Crawford, Robert J. Harrison, Shantenu Jha, Vijay S. Pande, and Teresa Head-Gordon, "Perspective: Computational chemistry software and its advancement as illustrated through three grand challenge cases for molecular science," Journal of Chemical Physics 149, 180901 (2018).

[46] Nancy Wilkins-Diehr and T. Daniel Crawford, "NSF's inaugural software institutes: The science gateways community institute and the molecular sciences software institute," Computing in Science \& Engineering 20, 26-38 (2018).

[47] http://cleanenergy.molecularspace.org (accessed July 3, 2013).

[48] http://www.worldcommunitygrid.org (accessed July 3, 2013). 